\title{
DNA methylation profiling unveils TGF- $\beta$ hyperresponse in tumor associated fibroblasts from lung cancer patients
}

Miguel Vizoso, Marta Puig, F. Javier Carmona, María Maqueda, Antonio Gomez, Anna Labernardie, Marta Gabasa, Saioa Mendizuri, Rafael Ikemori, Xavier Trepat, Sebastian Moran, Enrique Vidal, Noemí Reguart, Alexandre Perera, Manel Esteller and Jordi Alcaraz

\begin{abstract}
There is growing interest in defining the aberrant molecular differences between normal and tumor-associated fibroblasts (TAFs) that support tumor progression. For this purpose, we recently conducted a genome-wide DNA methylation profiling of TAFs and paired control fibroblasts (CFs) from non-small cell lung cancer (NSCLC) patients, and reported a widespread hypomethylation concomitantly with focal gain of DNA methylation; in addition, we found evidence that a fraction of lung TAFs are fibrocytes in origin. Of note, the aberrant epigenome of lung TAFs had a global impact in gene expression and a selective impact on the TGF- $\beta$ pathway. To get insights on the functional implications of the latter impact, we analyzed the response of lung TAFs to exogenous TGF- $\beta 1$ in terms of activation and contractility. We found a larger expression of a panel of activation markers including $\alpha$-SMA and collagen-I in TAFs compared to control fibroblasts. Likewise, TGF- $\beta 1$ elicited a larger contractility in TAFs than in CFs as assessed by traction force microscopy. These findings reveal that lung TAFs are hyperresponsive to TGF- $\beta 1$, which may underlie the expansion and/or maintenance of the tumor-promoting desmoplastic stroma in lung cancer.
\end{abstract}

